\title{
Image-based 3D non-rigid respiratory motion correction for free-breathing thoracic MR angiography
}

\author{
Fei Han ${ }^{1 *}$, Cheng Ouyang ${ }^{2}$, Ziwu Zhou', Paul J Finn ${ }^{1}$, Peng Hu${ }^{1}$ \\ From 19th Annual SCMR Scientific Sessions \\ Los Angeles, CA, USA. 27-30 January 2016
}

\section{Background}

Respiratory motion compensation is usually required in free-breathing thoracic MR imaging applications to remove the motion induced blur and ghosting artifacts. Most respiratory motion compensation techniques are based on gating in which data is acquired only at one respiratory state (RS) and therefore suffer from extended yet unpredictable scan time. Other techniques compensate for motion by correcting the phase of motion corrupted $\mathrm{k}$-space data so that data acquired in multiple respiratory states can be used in reconstruction for improved scan efficiency. However, these methods are often limited to correcting rigid-body motion and thus not suitable for thoracic imaging with large field-ofview. In this work, we propose a technical strategy of correcting 3D non-rigid motion and apply it to ferumoxytol enhanced free-breathing thoracic MRA.

\section{Methods}

In the proposed method, the non-rigid motion is modeled using voxel-based linear translation, which is estimated using 3D image registration. The workflow is listed in Fig. 1: 1) acquired data is binned into different RS based on a respiratory surrogate signal (e.g. navigator, self-gating). Dynamic k-space sampling pattern is required so that images can be reconstructed from each data bin; 2) quantitative motion estimation is performed between a chosen "reference bin" and one of the "correction bins" using 3D non-rigid image registration, generating a voxel-based 3D motion vector field. Motion vectors are then processed using $\mathrm{k}$-means clustering and represented by fewer approximations for reduced

Radiology, UCLA, Los Angeles, CA, USA

Full list of author information is available at the end of the article computational cost in the next step; 3) kspace linear phase corrections are performed using each motion vector on the "correction bin", which are then combined with the reference bin for reconstruction; 4) the final images are generated by pixel-based image fusion of all the motion corrected image candidate, where the selection is made based on the estimated motion vector in step 2.

The proposed method was applied on a ferumoxytolenhanced free-breathing thoracic MRA dataset acquired on a 1 year old patient under general anesthesia with mechanical ventilation. The data was retrospectively binned into 2 RS using airway pressure signal. The kspace under-sampling rate of each bin was $12 \mathrm{X}$. Other scan parameters includes: matrix $=500 \times 240 \times 120$; $1 \mathrm{~mm}^{3}$ resolution; 6 cardiac phases; TA $=6$ min. Image registration was performed using Elastix toolbox. Images were reconstructed using ESPIRiT.

\section{Results}

The motion corrected image has higher SNR (blood pool: 25.4 vs 14.5$)$ than the ones reconstructed from single RS because double amount of kspace data was used. When compared with the images from uncorrected 2 RS, vascular and cardiac structures in the chest and abdomen are better defined in the motion corrected images. (Fig. 1)

\section{Conclusions}

Our preliminary result shows that the proposed method could correct for complex respiratory motion in thoracic and abdomen imaging applications. The scan efficiency could be significantly increased since more k-space data is used. 


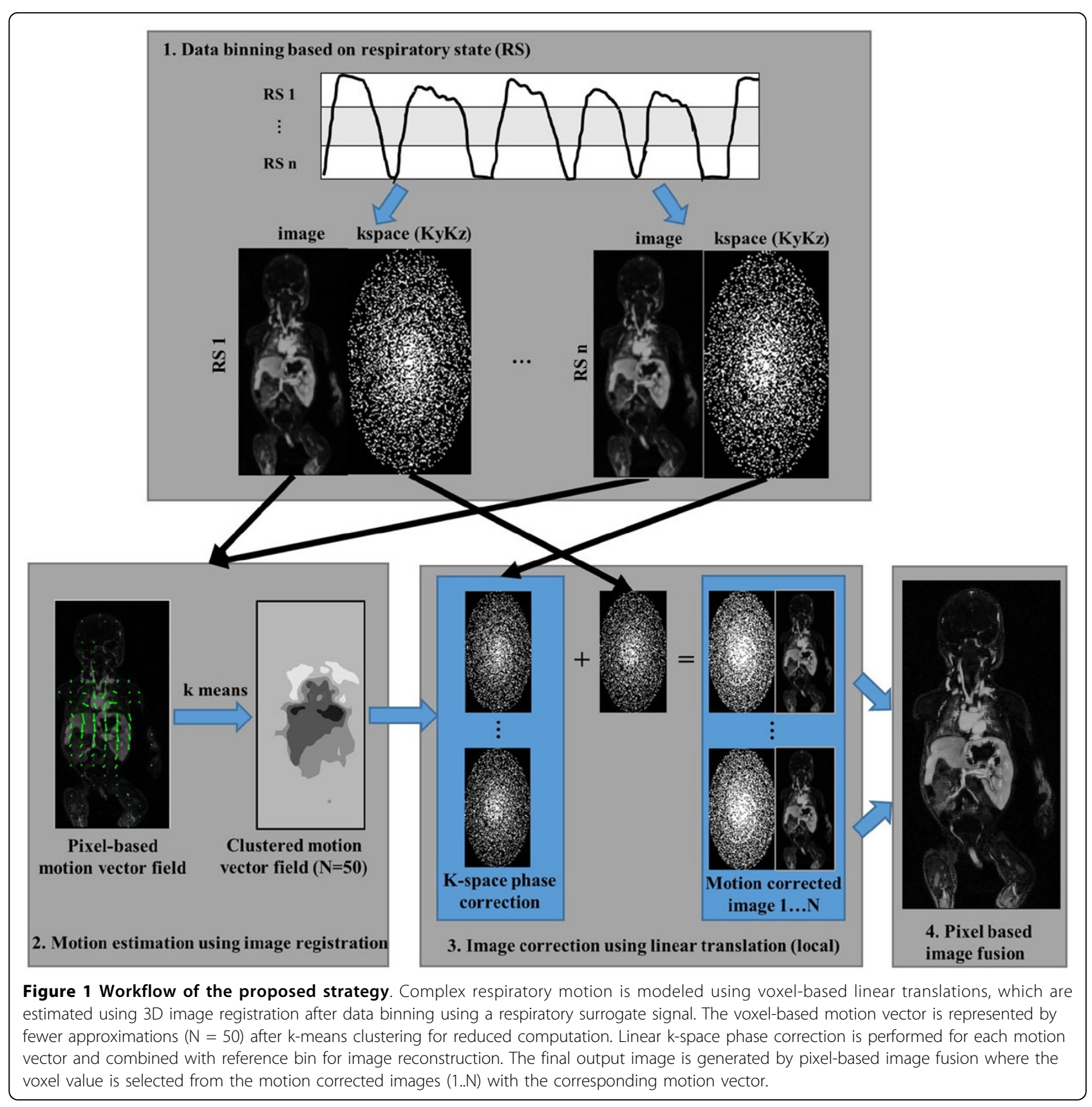




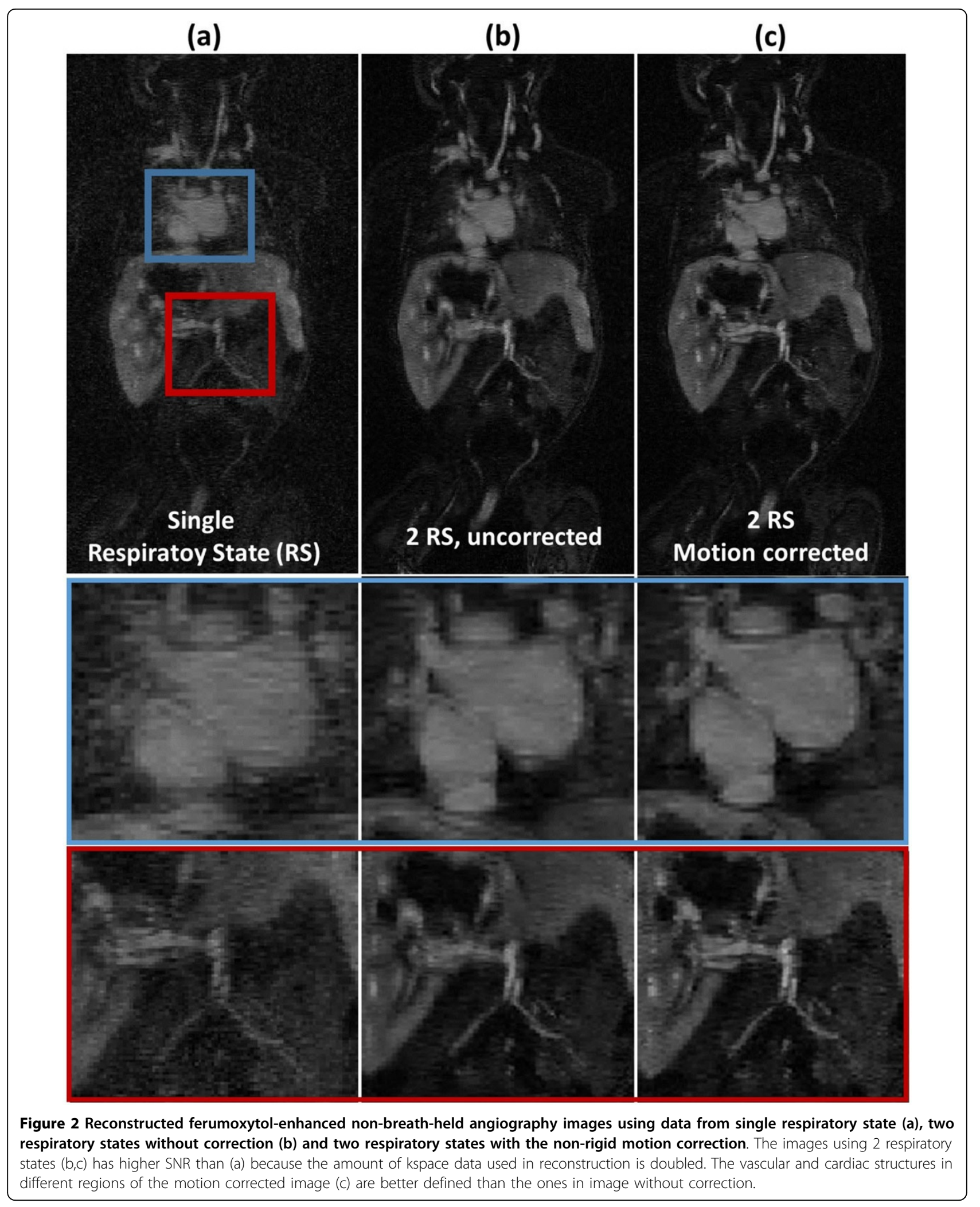




\section{Authors' details}

'Radiology, UCLA, Los Angeles, CA, USA. Biomedical Engineering, Xi'an

Jiaotong University, Xi'an, China.

Published: 27 January 2016

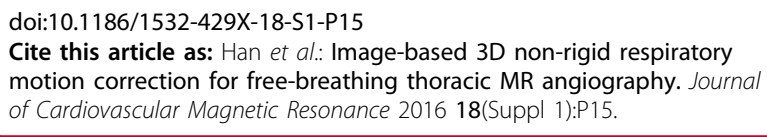

Submit your next manuscript to BioMed Central and take full advantage of:

- Convenient online submission

- Thorough peer review

- No space constraints or color figure charges

- Immediate publication on acceptance

- Inclusion in PubMed, CAS, Scopus and Google Scholar

- Research which is freely available for redistribution 